\title{
Multi-band optical micro-variability observations of BL Lacertae
}

\author{
I. E. Papadakis ${ }^{1,2}$, P. Boumis ${ }^{2, \star}$, V. Samaritakis ${ }^{2}$, and J. Papamastorakis ${ }^{2,1}$ \\ 1 IESL, Foundation for Research and Technology-Hellas, PO Box 1527, 71110 Heraklion, Crete, Greece \\ 2 Physics Department, University of Crete, PO Box 2208, 71003 Heraklion, Greece
}

Received 7 June 2002 / Accepted 24 October 2002

\begin{abstract}
We have observed BL Lacertae in the $B, R$ and $I$ bands for 2 nights in July, 1999, and 3 nights in July, 2001. The observations resulted in almost evenly sampled light curves, with an average sampling interval of $\sim 5$ min. Because of the dense sampling and the availability of light curves in three bands we are able to study the intra-night flux and spectral variability of the source in detail. The source is significantly variable in all bands, showing variations on different time scales. On average, the variability amplitude increases from $\sim 5 \%$ in the $I$ band, to $\sim 5.5 \%$ in the $R$ and $\sim 6.5 \%$ in the $B$ band light curves. The rising and decaying time scales are comparable within each band, but they increase from the $B$, to $R$ and $I$ band light curves. The optical power spectrum shows a red noise component with a slope of $\sim-2$. Cross-correlation analysis shows that in most cases the delay between the variations in the $B$ and $I$ band light curves is less than $\sim \pm 0.4 \mathrm{hrs}$. However, the cross-correlation functions are asymmetric, implying complex delays of the $I$ band variations with respect to the $B$ band variations. Furthermore, in one case we find that the $I$ band variations are significantly delayed (by $\sim 0.2 \mathrm{hrs}$ ) with respect to the $B$ band variations. We also detect significant spectral variations. We find that the spectrum hardens, (i.e. it gets flatter) as the flux increases, and the flattest spectral index corresponds to the maximum $B$ band flux. The rate of the spectral variations does not remain the same during the observations. Our results imply that the fast, intra-night variations of the source correspond to perturbations of different regions in the jet which cause localized injections of relativistic particles on time scales much shorter that the average sampling interval of the light curves. The variations are controlled by the cooling and light crossing time scales, which are probably comparable.
\end{abstract}

Key words. galaxies: active - galaxies: BL Lacertae objects: general - galaxies: BL Lacertae objects: individual: BL Lacertae galaxies: jets

\section{Introduction}

BL Lac objects are one of the most peculiar classes of active galactic nuclei (AGN). They show high polarization (up to a few percent, as opposed to less than $\sim 1 \%$ for most AGNs) and usually do not exhibit strong emission or absorption lines in their spectra (but see for example Corbett et al. 1996, and Vermeulen et al. 1995 for BL Lac itself, and Pian et al. 2002, for PKS 0537-441). They also show continuum variability at all wavelengths at which they have been observed, from Xrays to radio wavelengths. In the optical band they show large amplitude, short-time scale variations. The overall spectral energy distribution of BL Lacs shows two distinct components in the $v-v F_{v}$ representation. The first one peaks from $\mathrm{mm}$ to the $\mathrm{X}$-rays, while the second component peaks at GeV-TeV energies (e.g. Fossati et al. 1998). The commonly accepted scenario assumes that the non-thermal emission from BL Lacs is

Send offprint requests to: I. E. Papadakis,

e-mail: jhep@physics.uoc.gr

* Present address: Institute of Astronomy \& Astrophysics, National Observatory of Athens, I. Metaxa \& V. Paulou, P. Penteli, 15236, Athens, Greece. synchrotron and inverse-Compton radiation produced by relativistic electrons in a jet oriented close to the line of sight (e.g. Ghisellini et al. 1998).

BL Lacertae, the object that was used to define this class of AGN, is a well-studied source that has been observed in the optical band for more than a century. Large amplitude variations have been observed on both long and short time scales (Villata et al. 2002, and references therein). BL Lac was among the first BL Lac objects which showed large amplitude variations on time scales of $\sim$ hours (Racine 1970; Miller et al. 1989). During the long, large amplitude 1997 optical outburst the intra-night variations of the object were studied in detail. Nesci et al. (1998) and Speziali \& Natali (1998) presented multi-band, micro-variability studies based on observations that were made in July 1997 and August 1997, respectively. In both cases, rapid variations were detected in all bands, with their amplitude increasing towards smaller wavelengths. Matsumoto et al. (1999), presented $V$ band observations of BL Lac during August and September, 1997, and reported the detection of a rapid flux increase of about 0.6 mag within $40 \mathrm{~min}$. Ghosh et al. (2000), presented $V$ band observations taken between August and October, 1997. They combined the 
optical fast, intra-night variations with simultaneous $\mathrm{X}$-ray and $\gamma$-ray observations, and analyzed their results within the context of theoretical models. Clements \& Carini (2001) presented $V$ and $R$ micro-variability observations that were obtained during the summer of 1997. They observed significant intra-night variations, which were associated with spectral variations as well. The spectrum of the source was becoming "bluer" as it brightened. Finally, Ravasio et al. (2002) presented simultaneous X-ray (from Beppo-Sax) and optical observations of the source during June and December, 1999. The source has varied continuously in the optical band. No clear correlation between fast X-ray and optical variability could be found.

In this work, we present simultaneous, $B, R$ and $I$ band monitoring, intra-night observations of BL Lac obtained during 1999 and 2001. The availability of observations in different bands and the almost evenly, dense sampling pattern of the light curves offer us the opportunity to study in detail the flux and spectral variations of the source on time scales of $\sim \min$ to hours. The paper is organized as follows. In the next section we present our observations. In Sect. 3 we estimate the variability amplitude and compare the observed time scales between the different band light curves. In Sect. 4 we present the results from a power spectrum analysis, in Sect. 5 we discuss the spectral variability properties of the source, and in Sect. 6 we present the cross-correlation analysis results. A discussion follows in Sect. 7, while a summary of our work is presented in Sect. 8 .

\section{Observations and data reduction}

BL Lac was observed for 2 nights in 1999 and 3 nights in 2001 from the $1.3 \mathrm{~m}, \mathrm{f} / 7.7$ Ritchey-Cretien telescope at Skinakas Observatory in Crete, Greece. The observations were carried out through the standard Johnson $B$ and Cousins $R, I$ filters. The CCD used was a $1024 \times 1024$ SITe chip with a $24 \mu \mathrm{m}^{2}$ pixel size (corresponding to $0 . ' 5$ on sky). The exposure time was 180,60 and $30 \mathrm{~s}$ for the $B, R$ and $I$ filters, respectively, during the 2001 observing run, and 120, 60 and $30 \mathrm{~s}$, respectively, during the 1999 run. In Table 1 we list the observation dates, and the number of the $B, R$ and $I$ frames that we obtained each night. During the observations, the seeing was between $\sim 1^{\prime \prime}-2$ ". Standard image processing (bias subtraction and flat fielding using twilight-sky exposures) was applied to all frames.

We performed aperture photometry of BL Lac and of the comparison stars $\mathrm{B}, \mathrm{C}, \mathrm{H}$, and $\mathrm{K}$ by integrating counts within a circular aperture of radius $10^{\prime \prime}$ centered on the objects. The photometry of the comparison stars was taken from Smith et al. (1985) and Fiorucci \& Tosti (1996) for the $B$ and the $R, I$ bands, respectively. The calibrated magnitudes of BL Lac were corrected for reddening and the contribution of the host galaxy as follows.

For the reddening correction of the nuclear fluxes, we used the relationship: $A_{V}=N_{H} / 2.23 \times 10^{21}$ (Ryter 1996), in order to estimate the $V$-band extinction ( $A_{V}$, in magnitudes). We assumed the column density value of $N_{H}=2.0 \times 10^{21} \mathrm{~cm}^{-2}$, derived from the $\mathrm{X}$-ray measurements with ROSAT (Urry et al. 1996). This value should be representative of the nuclear flux
Table 1. Date of observations, and number of frames (nof) obtained at each band.

\begin{tabular}{lccc}
\hline \hline Date & $\begin{array}{c}B \\
\text { (nof) }\end{array}$ & $\begin{array}{c}R \\
\text { (nof) }\end{array}$ & $\begin{array}{c}I \\
\text { (nof) }\end{array}$ \\
\hline $28 / 07 / 99$ & 39 & 39 & 39 \\
$29 / 07 / 99$ & 37 & 37 & 37 \\
$05 / 07 / 01$ & 40 & 37 & 38 \\
$06 / 07 / 01$ & 38 & 38 & 38 \\
$08 / 07 / 01$ & 42 & 44 & 42 \\
\hline
\end{tabular}

absorption, and its use implies $A_{V}=0.907$. Using the $A_{\lambda}$ versus $\lambda$ relationship of Cardelli et al. (1989) we found the extinction (in magnitudes) in the $B, R$, and $I$ filters: $A_{B}=1.21, A_{R}=0.73$, and $A_{I}=0.54$

We converted the dereddened magnitudes into flux, and then we corrected for the contribution of the host galaxy to the measured flux in each band. According to Scarpa et al. (2000), the $R$ band magnitude of the BL Lac host galaxy is $R_{\text {host }}=15.55 \pm 0.02$. In order to correct $R_{\text {host }}$ for reddening, we used the value of $A_{R}=0.88$ (taken from NED ${ }^{1}$ ). This value is derived from the Schlegel et al. (1998) maps, and may be more representative of the Galactic absorption for the host galaxy, which is more extended than the active nucleus itself. We inferred the $B_{\text {host }}$ and $I_{\text {host }}$ magnitudes adopting the elliptical galaxy colors (at redshift zero) of $V-R=0.61, B-V=0.96$, and $R-I=0.70$ (Fukugita et al. 1995). The resulting host galaxy fluxes in the $B, R$, and $I$ bands are $1.40,4.29$, and $6.76 \mathrm{mJy}$, respectively. Using the results of Scarpa et al. (2000) and a de Vaucouleurs $r^{1 / 4}$ profile, we estimated that the host galaxy contribution within the circular aperture of radius $10^{\prime \prime}$ centered on BL Lac should be $70 \%$ of the whole galaxy flux. Therefore, the final $B_{\text {host }}, R_{\text {host }}$, and $I_{\text {host }}$ fluxes that were subtracted from the observed BL Lac fluxes were 0.98,3.0, and $4.73 \mathrm{mJy}$, respectively.

\section{The observed light curves}

In Fig. 1 we show the final $B, R$, and $I$ light curves of BL Lac during our observations. In the same figure we also show the $B$ band light curve of the comparison star $B$. While the light curve of this star (and of the other 3 comparison stars in all bands) does not show significant variations, significant intranight variations can be observed in the BL Lac light curves at all bands. During the July 28 and 29, 1999 observations we detected two flare-like events at the beginning and at the end of the observations, respectively. During the July 5 and 8, 2001 observations the flux decreased at the beginning of the night and then, after 2-4 hours, it increased again. Finally, a well defined flare which lasted for $\sim 3$ hours was detected in July 6 , 2001. Overall, the observed light curves show smooth variations which last for a few hours, with no significant variations on time scales of $\sim$ minutes.

1 The NASA/IPAC Extragalactic Database (NED) is operated by the Jet Propulsion Laboratory, California Institute of Technology, under contract with the National Aeronautics and Space Administration. 


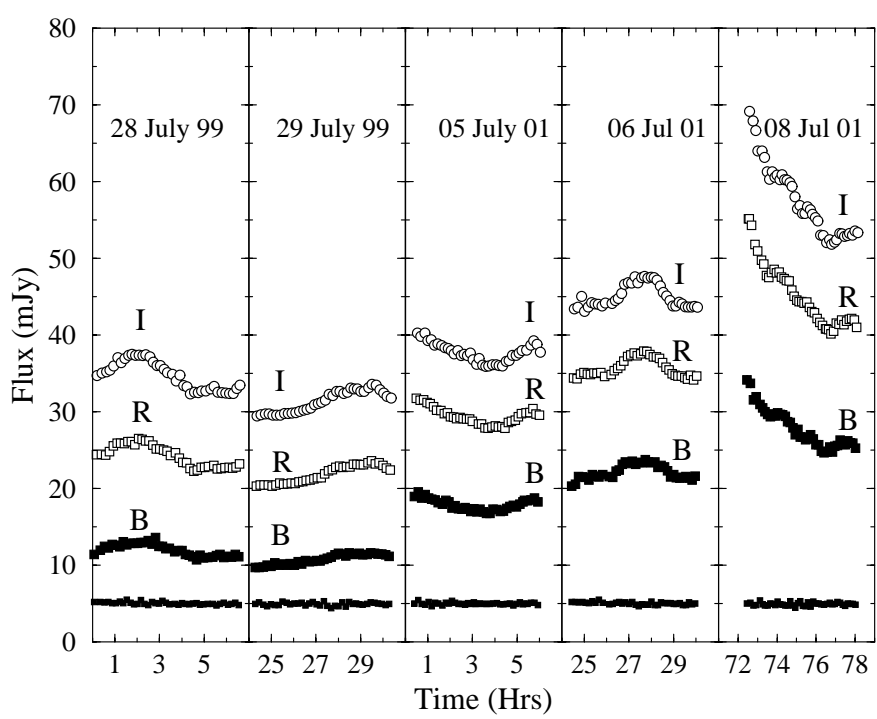

Fig. 1. $B, R$, and $I$ band light curves during the 1999 and 2001 observations. Errors are also plotted but are smaller than the size of the light curve points. Time is measured in hours from 20:00 UT on July 5, 2001, for the 2001 observations, and from 19:00 UT on July 28, 1999, for the 1999 observations. The small filled squares on the bottom of the figure show the $B$ band light curve of the comparison star B. (For clarity reasons, the light curve is shifted from a mean of $\sim 7 \mathrm{mJy}$ to $5 \mathrm{mJy}$.)

Table 2. The fractional variability amplitude of the light curves at each band.

\begin{tabular}{lccc}
\hline \hline Date & $\begin{array}{c}f_{\mathrm{rms}, B} \\
(\%)\end{array}$ & $\begin{array}{c}f_{\mathrm{rms}, R} \\
(\%)\end{array}$ & $\begin{array}{c}f_{\text {rms }, I} \\
(\%)\end{array}$ \\
\hline $28 / 07 / 99$ & $7.2 \pm 0.7$ & $6.2 \pm 0.4$ & $5.7 \pm 0.3$ \\
$29 / 07 / 99$ & $6.9 \pm 0.7$ & $5.5 \pm 0.3$ & $4.8 \pm 0.3$ \\
$05 / 07 / 01$ & $4.2 \pm 0.4$ & $3.9 \pm 0.3$ & $3.5 \pm 0.3$ \\
$06 / 07 / 01$ & $4.3 \pm 0.4$ & $3.6 \pm 0.2$ & $3.6 \pm 0.2$ \\
$08 / 07 / 01$ & $9.1 \pm 0.8$ & $8.8 \pm 0.7$ & $8.5 \pm 0.6$ \\
\hline
\end{tabular}

In order to compare the amplitude of the variations that we observe in the different band light curves, we computed the "fractional variability amplitude" $\left(f_{\mathrm{rms}}\right)$ of all the light curves. The fractional variability amplitude is defined as: $f_{\mathrm{rms}}=$ $\left(\sigma^{2}-\sigma_{N}^{2}\right)^{1 / 2} / \bar{x}$, where $\sigma^{2}$ is the sample variance of the light curve, $\sigma_{N}^{2}=\sum_{i=1}^{N} e r r_{i}^{2} / N$ is the variance introduced by the instrumental noise process (err $r_{i}$ is the error associated with each point, $i$, in the light curve), and $\bar{x}$ is the light curve mean. The fractional variability amplitude represents the average amplitude of the observed variations as a percentage of the light curve mean. The $f_{\mathrm{rms}}$ values are listed in Table 2. The errors on the $f_{\text {rms }}$ values were estimated using the bootstrap method of Peterson et al. (1998). They represent the uncertainty associated with the flux uncertainties in the individual measurements and the uncertainty associated with the observational sampling of the light curves (i.e. the size of the intervals between observations and the length of the light curves). On average, the amplitude of the observed variations is similar for both the 1999 and 2001 observations. Furthermore, the variability amplitude increases from the $I$ to the $B$ band. The average $f_{\mathrm{rms}}$ of the $B, R$,

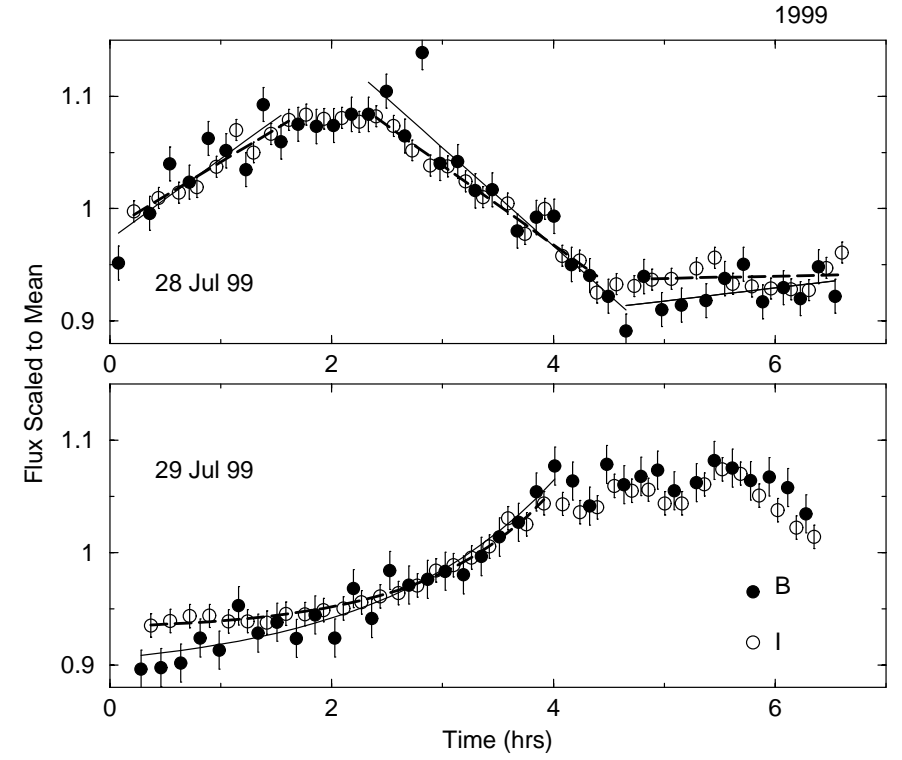

Fig. 2. $B$ and $I$ band light curves in 1999 (filled and open circles, respectively). The solid and dashed lines show the best fitting model to the "rising" and "decaying" parts of the $B$ and $I$ light curves, respectively. Time is measured in hours from the beginning of the observations at each night.

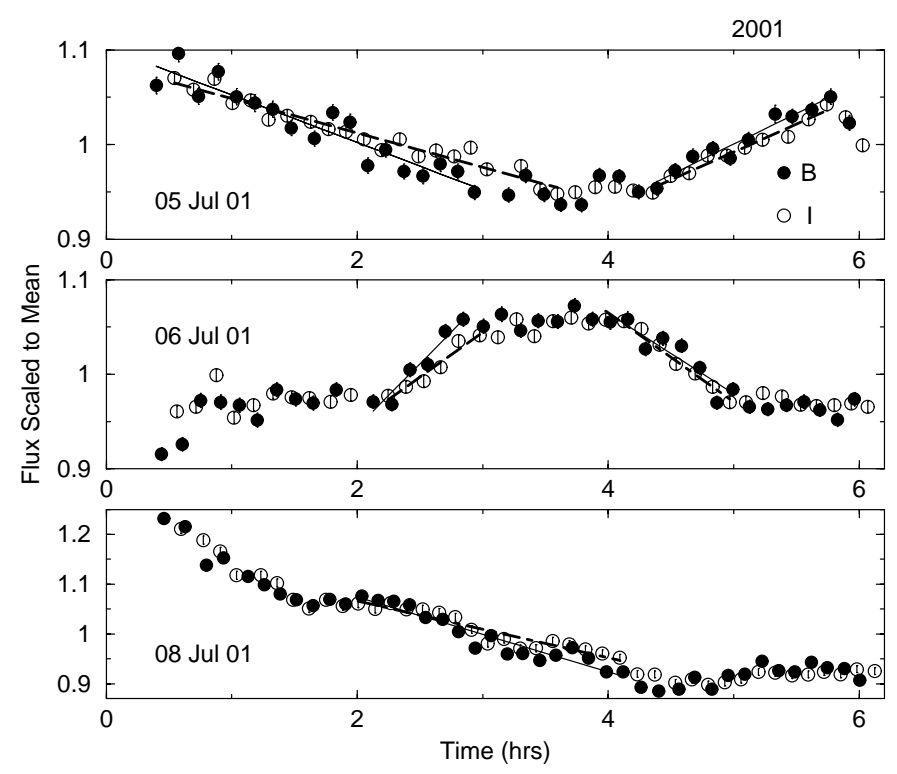

Fig. 3. Same as Fig. 2, for the 2001 light curves.

and $I$ light curves is $6.3 \pm 0.3 \%, 5.6 \pm 0.2 \%$, and $5.2 \pm 0.2 \%$, respectively.

In order to visualize how well the light curves in the three bands agree with each other, we normalized them to their mean, and plotted them together (Figs. 2 and 3 for the 1999 and 2001 light curves, respectively; for clarity reasons we have plotted only the $B$ and $I$ band light curves). These plots show that the light curves are well correlated. They also show that the "rising"/“decaying" parts of the $B$ band light curves are steeper than the respective parts in the $I$ band light curves.

In order to compare the variability time scales between the $B, R$, and $I$ band light curves, we estimated their "Flux 
Table 3. The "Flux Variability Rate" $\left(F V R\right.$, in units of $\left.\times 10^{-2} \mathrm{mJy} / \mathrm{hr}\right)$ during the rising and decaying parts of the $B$ and $I$ band light curves.

\begin{tabular}{lcccc}
\hline \hline Date & $\begin{array}{c}F V R_{B} \\
\text { (rise) }\end{array}$ & $\begin{array}{c}F V R_{B} \\
\text { (decay) }\end{array}$ & $\begin{array}{c}F V R_{I} \\
\text { (rise) }\end{array}$ & $\begin{array}{c}F V R_{I} \\
\text { (decay) }\end{array}$ \\
\hline $28 / 07 / 99$ & $7.1 \pm 1.7$ & $-8.7 \pm 0.9$ & $6.0 \pm 0.7$ & $-7.1 \pm 0.5$ \\
$29 / 07 / 99$ & $4.1 \pm 0.4$ & - & $3.1 \pm 0.3$ & - \\
$05 / 07 / 01$ & $6.5 \pm 0.5$ & $-5.0 \pm 0.4$ & $5.9 \pm 0.3$ & $-3.6 \pm 0.2$ \\
$06 / 07 / 01$ & $13.4 \pm 1.7$ & $-8.5 \pm 1.5$ & $9.5 \pm 0.1$ & $-9.4 \pm 0.6$ \\
$08 / 07 / 01$ & - & $-7.5 \pm 0.6$ & - & $-5.7 \pm 0.5$ \\
\hline
\end{tabular}

Variability Rate" $(F V R)$ as follows. First, we identified the rising and decaying parts of the light curves. Then, we fitted them with a linear model of the form: $\operatorname{flux}(t)=A+B \times t$ (where $t$ is time in hours). The linear model fits well the rising/decaying phases in all the light curves (as shown by the solid and dashed lines in Figs. 2 and 3), except for the rising part of the July 29, 1999 light curves, which was best fitted by an exponential function of the form: $f l u x(t)=A_{1} \times \exp (t / \tau)+A_{2}$. Based on the best model fitting results, we estimated the $F V R$ (in units of $\mathrm{mJy} / \mathrm{hrs}$ ) for the different phases of each light curve. In effect, the $F V R$ values show the amount of the flux variation per unit time. The results for the $B$ and $I$ band light curves are listed in Table 3 (similar model fitting results were also obtained for the $R$ band light curves as well).

The average $B$ and $I$ band $F V R$ values are: $F \bar{V} R_{B \text {,rise }}=$ $7.8 \pm 0.6, F \bar{V} R_{B, \text { decay }}=-7.4 \pm 0.5$, and $F \bar{V} R_{I, \text { rise }}=6.1 \pm$ $0.3, F \bar{V} R_{I, \text { decay }}=-6.4 \pm 0.2$ (all values are in units of $\times 10^{-2} \mathrm{mJy} / \mathrm{hr}$ ). These results show that the rising $F V R$ values are similar, in absolute magnitude, to the decaying $F V R$ values, in both the $B$ and $I$ bands. This result implies that the rising and decaying parts of the light curves are symmetric. However, when we compare the values between the two bands we find that the average $F V R_{B}$ values are larger (in absolute magnitude) than the $F V R_{I}$ band values: $F \bar{V} R_{B \text {,rise }}-F \bar{V} R_{I, \text { rise }}=1.7 \pm$ $0.8 \times 10^{-2} \mathrm{mJy} / \mathrm{hr}$, and $F \bar{V} R_{B \text {,decay }}-F \bar{V} R_{I \text {, decay }}=-1.0 \pm 0.5$ $\times 10^{-2} \mathrm{mJy} / \mathrm{hr}$. Therefore, the rising/decaying time scales in the $B$ band are shorter than in the $I$ band. However this difference is significant only at the $2 \sigma$ level.

Finally, apart from the rising/decaying parts, we can identify at least two flares with a broad peak during the July 6, 2001 and July 28, 1999 observations. This "flare plateau" state lasted for $\sim 1 \mathrm{hr}$ in both cases and in all light curves. It is possible that the last part of the July 29, 1999 observation may also represent the plateau state of yet another flare. However, we cannot be certain because of the lack of observations of the decaying phase of the flare.

\section{Power spectrum analysis}

In order to quantify the variability seen in the optical light curves of BL Lac we estimated their power spectrum as follows. For each individual light curve, we computed the periodogram as:

$\hat{I}\left(v_{i}\right)=(\Delta t / N)\left\{\sum_{i=1}^{N}\left[x\left(t_{i}\right) / \bar{x}-1\right] \mathrm{e}^{-i 2 \pi v_{i} t_{i}}\right\}^{2}$,
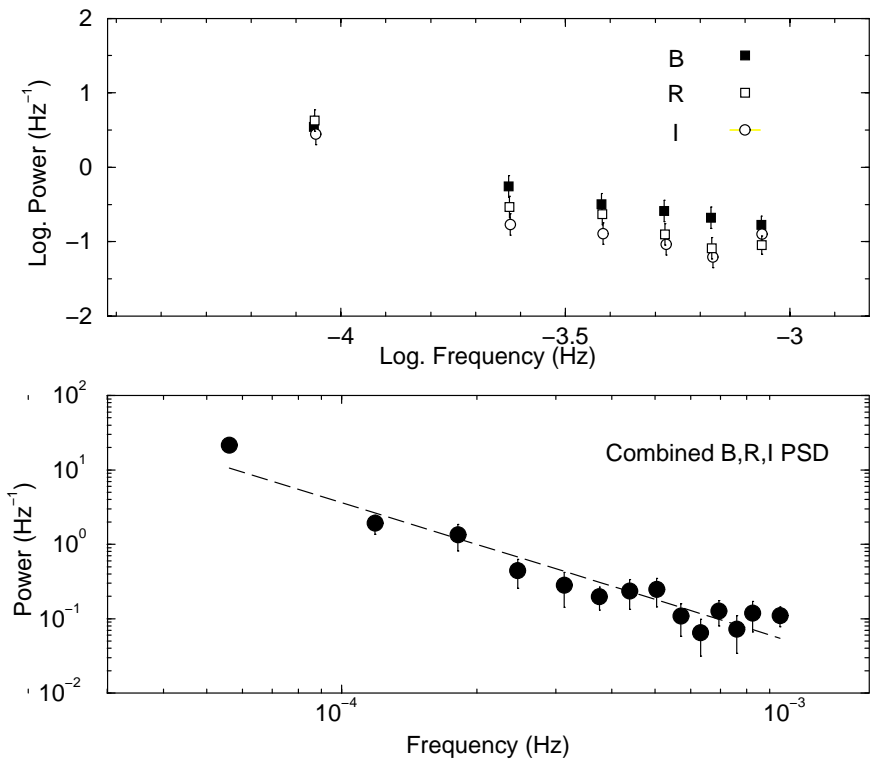

Fig. 4. Average $B, R$, and $I$ band power spectra of BL Lac (upper panel). In the lower panel, we plot the "average optical power spectrum" of the source, with the experimental noise component subtracted. The dashed line shows the best fitting power law model to the data.

where $\bar{x}, \Delta t$, and $N$ are the mean value, bin size, and number of points of each light curve, respectively, and $v_{i}=i /(N \Delta t)$, $i=1,2, \ldots,(N / 2)-1$ (e.g. Papadakis \& Lawrence 1993). The periodogram calculated in this way (i.e. with the points normalized to the light curve mean) has the units of $\mathrm{Hz}^{-1}$. This normalization is necessary in order to combine the periodograms of the different light curves (see below). As $\Delta t$ we accepted the mean interval between the points in each light curve. We note that, due to the continuous monitoring of the source, the light curves are almost evenly sampled. The intervals between successive points are almost equal, and any minor difference cannot affect seriously the estimation of the power spectrum. Finally, we combined the periodograms of all the light curves in each band, we sorted them in order of increasing frequency, we computed their logarithm and grouped them into bins of size 15 following the method of Papadakis \& Lawrence (1993).

The resulting $B, R$, and $I$ band power spectra are shown in the upper plot of Fig. 4 (filled squares, open squares and open circles, respectively). They all show a similar red noise component, i.e. they all increase logarithmically towards lower frequencies. The power spectrum normalization increases slightly from the $I$ to the $B$ band power spectrum, as expected from the fact that the $B$ band light curves show the largest $f_{\text {rms }}$ values.

Since the three power spectra show a similar shape, we combined the periodograms of all the light curves in order to estimate an "average optical band" power spectrum of the source. Since the Poisson noise power level is different in each light curve (due to the fact that the experimental errors are different), we subtracted the expected Poisson noise level from each periodogram, we sorted them in order of increasing frequency, and grouped the periodogram estimates into bins of size 20 . 


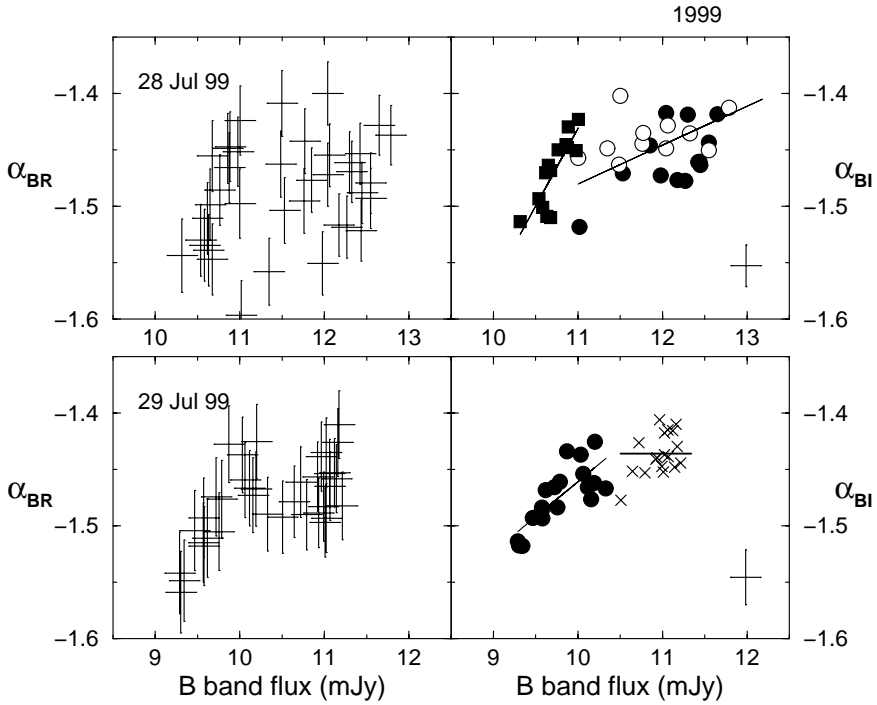

Fig. 5. $B-R$ and $B-I$ spectral indices vs. the $B$ band source flux (left and right panels, respectively) for the 1999 observations. The different symbols for the $B-I$ vs. $B$ data in each plot correspond to different parts of the light curves as explained in the text. The crosses in the $\left[B, \alpha_{B I}\right]$ plots indicate the average error of the points in the plots.

The average optical power spectrum of BL Lac is plotted in the lower plot of Fig. 4. Using standard $\chi^{2}$ statistics, we fitted the power spectrum with a power law model of the form $P(v)=$ $A v^{-a}$. The model provides a good fit to the data $\left(\chi^{2}=10.6\right.$ for 12 d.o.f.). The best fitting model is also plotted in Fig. 4 (dashed line). The best fitting slope value is $a=1.87 \pm 0.16$ (all errors quoted in the paper correspond to the $68 \%$ confidence region).

\section{Spectral variability}

Since $f_{\mathrm{rms}}$ is different in the $B, R$, and $I$ band light curves, and there is an indication that the rise/decay time scales may be different as well, we expect the flux variations to be associated with spectral variations. In order to investigate this possibility we used the dereddened light curves, after correction for the host galaxy contribution, to calculate the two-point spectral indices $\left(B-R, \alpha_{B R}\right.$, and $\left.B-I, \alpha_{R I}\right)$ using the equation, $\alpha_{12}=\log \left(F_{1} / F_{2}\right) / \log \left(v_{1} / v_{2}\right)$, where $F_{1}$ and $F_{2}$ are the flux densities at frequencies $v_{1}$ and $v_{2}$, respectively.

Figures 5 and 6 show the $\alpha_{B R}$ and $\alpha_{B I}$ versus the $B$ band flux plots for the 1999 and 2001 observations, respectively. The $\chi^{2}$ values for a constant slope show that the variations are statistically significant. The $\alpha_{B R}$ and $\alpha_{B I}$ variations are broadly correlated with the source flux in a similar way. Overall, as the $B$ band flux increases, both $\alpha_{B R}$ and $\alpha_{B I}$ increase as well. This result implies that the spectrum becomes "harder" (i.e. "bluer") as the flux increases. However, the relation between flux and spectral index is not simple. For example, a linear model of the form $\alpha=A+B \times$ flux, cannot fit well any of the $\left[B, \alpha_{B I}\right]$ plots in Figs. 5 and 6 (except for the $\left[B, \alpha_{B I}\right]$ plot of the July 29, 1999 observations). Because of this reason, we considered the spectral slope variations during the different phases of the light curves (i.e. rising, decaying, plateau) and we present our results below. We focus our discussion on the $\left[B, \alpha_{B I}\right]$ plots mainly,

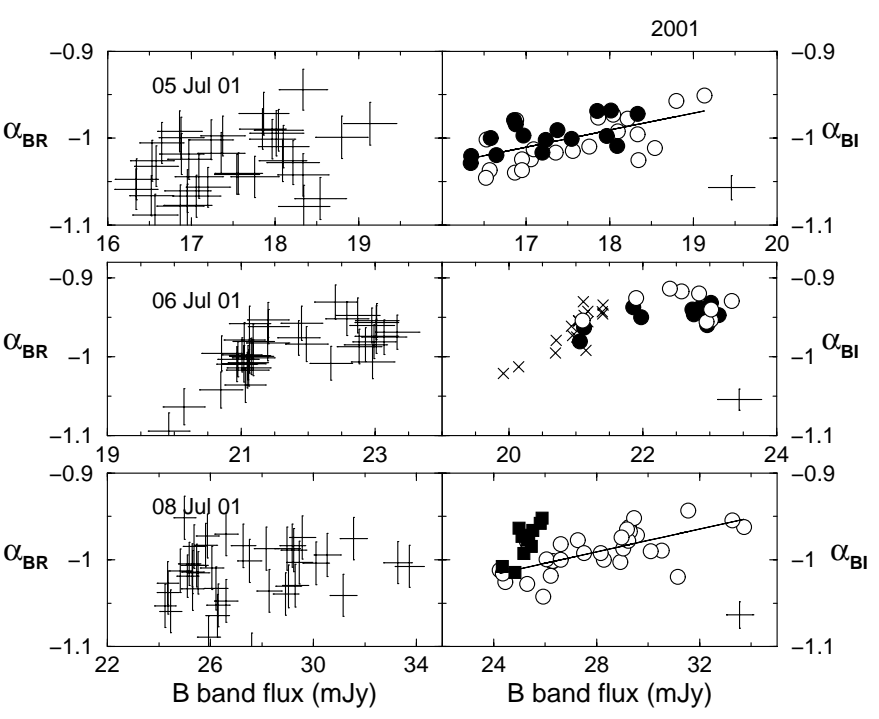

Fig. 6. Same as Fig. 5, for the 2001 observations.

since the errors on the $\alpha_{B I}$ points are smaller than the errors of the $\alpha_{B R}$ points.

During the July 28, 1999 observations, the flux increased at the beginning of the observations, it reached a plateau, then it decreased, and rose again (at a slower rate) towards the end of the observation (see Figs. 1 and 2). During the rising part of the flare, the spectral slope increased (solid circles in the upper right plot of Fig. 5), it reached its maximum value during the plateau state, and then decreased again (open circles in the same plot) as the source flux decreased. The flux related slope variations in the two parts of the flare follow the same linear trend (shown with a solid line in the same plot of Fig. 5). During the last part of the observation, the moderate flux increase is associated with a marked spectral slope increase (shown with solid squares). This is caused by the fact that the $B$ band flux increases faster than the $I$ band flux. During the rising part of the July 29, 1999 observation the spectral index increases linearly with the source flux (solid circles in the lower right plot in Fig. 5; the best fitting linear model is also shown with the solid line). It reaches its maximum value during the plateau state (shown with crosses in the same plot), which lasts until the end of the observation.

A linear relation between spectral slope and source flux is also observed during the July 5, 2001 observation (upper right plot, Fig. 6). As the flux decreased during the first part of the observation, the spectral slope decreased as well (open circles in this plot). Then, when the flux increased during the second part of the observation, the spectral slope also increased (solid circles). The linear trend is similar for both the rise/decay parts of the observation, although the normalization is different, with the rising part resulting in systematically larger spectral slope values. A similar behaviour is observed during the July 8, 2001 observation. During the first, long, decaying part of the observation, the spectral slope decreased (open circles, bottom plot in Fig. 6). A linear model describes well the overall trend (shown with solid line in the same plot), but it cannot fit well the data. This is mainly due to the fact that a few, small amplitude sub-flares can be seen on top of the long, decreasing phase 


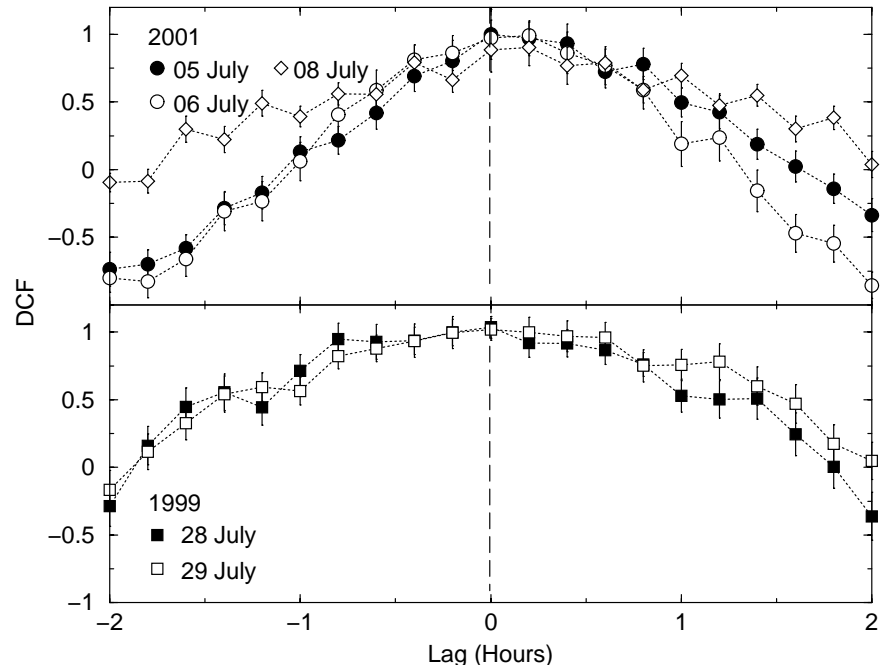

Fig. 7. Cross-correlation functions between the $B$ band and $I$ band light curves during the 1999 and 2001 observations (lower and upper plots, respectively).

of the light curves. The moderate flux increase at the end of the observation is associated with a steep spectral slope increase (shown with solid squares in the plot). This behaviour is similar to what was observed at the end of the July 28, 1999 observation. Finally, during the July 6, 2001 observations, the spectral slope remained constant $\left(\alpha_{B I} \sim-0.97\right.$, crosses in the middle plot of Fig. 6) before and after the flare which started two hours after the beginning of the observations. The spectral slope increased/decreased together with the flux in the rise/decay flare phases (shown with solid and open circles, respectively). The spectral slope reached its maximum value during the plateau phase.

\section{Cross-correlation analysis}

In order to compare the cross-links between the observed variations in the different bands we estimated the cross-correlation function (CCF) using the Discrete Correlation Function (DCF) of Edelson \& Krolik (1988). Figure 7 shows the DCF between the $B$ and $I$ band light curves during the 1999 and 2001 observations (lower and upper plot, respectively). In these plots, a positive lag means that the $B$ band leads the $I$ band variations. In order to quantify the maximum cross-correlation $\left(C C F_{\max }\right)$ and the "delay" between the light curves (i.e. the time lag, say $k_{\max }$, at which this maximum occurs) we fitted the DCF points around zero lag with a parabola, and accepted the best fitting values of the parabola peak as our best estimate of $C C F_{\max }$ and $k_{\max }$. Our results are listed in Table 4 (Cols. 2 and 3 for $C C F_{\max }$ and $k_{\max }$, respectively). The uncertainties in the observed lag values were estimated using the Monte Carlo simulation techniques of Peterson et al. (1998).

The DCF plots in Fig. 7 and the results on $C C F_{\max }$ show that the $B$ and $I$ band light curves are highly correlated, as expected from the visual good agreement between the different band light curves (e.g. Figs. 2 and 3). The estimated lag values $\left(k_{\max }\right)$ imply that the delay between the two band light curves is consistent with zero, within the errors. Using the
Table 4. The cross-correlation analysis results.

\begin{tabular}{lcc}
\hline \hline Date & $C C F_{\max }$ & $k_{\max }(\mathrm{hrs})$ \\
\hline $28 / 07 / 99$ & 1.0 & $-0.07 \pm 0.20$ \\
$29 / 07 / 99$ & 1.0 & $+0.10 \pm 0.28$ \\
$05 / 07 / 01$ & 0.90 & $+0.23_{-0.15}^{+0.19}$ \\
$06 / 07 / 01$ & 0.96 & $+0.09_{-0.12}^{+0.15}$ \\
$08 / 07 / 01$ & 0.76 & $+0.17_{-0.26}^{+0.29}$ \\
\hline
\end{tabular}

cross-correlation results from all the light curves (except for the July 5, 2001; see below), we estimate an average lag of $k_{\max }=0.07 \mathrm{hrs}$, while the $90 \%$ confidence limits are $\left|k_{\max }\right|<$ $0.42 \mathrm{hrs}$ (which is $\sim 4$ times the mean sampling rate of the light curves).

The CCF of the July 5, 2001 light curves, shows an indication that the $I$ band light curve is delayed with respect to the $B$ band light curve. We find a delay of $k_{\max }=0.23 \mathrm{hrs}(\sim$ twice the mean sampling interval of the light curves) with a $68 \%$ confidence region of [0.08-0.42 hrs]. In fact, the probability that the $I$ band is delayed with respect to the $B$ band light curve (i.e. the probability that $k_{\max }>0$ ) is $95.4 \%$.

Finally, all cross-correlation functions appear to be asymmetric (except for the July 28, 1999 CCF). The asymmetry is in the sense that, on time scales larger than 0.5 hours, the correlation at positive lags is larger than the correlation at negative lags. In fact, at positive lags, the $B$ band light curves are better correlated with the $I$ band light curves than with themselves. This result implies that the variability components with periods larger than $\sim 0.5$ hours in the $B$ band light curves lead the respective components in the $I$ band light curves.

\section{Discussion}

Although we cannot estimate systematically time scales from the data presented in this work, the fact that the duration of the rising/decaying parts of the light curves is different during our observations suggests the presence of different variability time scales in BL Lac. For example, we observe a flare which lasted for $\sim 3$ hours during the July 6, 2001 observations, while longer flares are detected during the July 28, and July 29, 1999 observations. Furthermore, the smooth, "long"-term trends observed during the July 5, and 8, 2001 observations, are probably parts of even longer flare-like events. If the difference in time scales correspond to differences in the size of the emitting source, then perhaps physically different regions/parts of the jet contribute to the optical emission of BL Lac. Even if this is the case, the similarity in the flux and spectral variability properties suggests that the same physical mechanism operates in all cases.

The rapid, optical variability properties of BL Lac are similar to the $X$-ray variability properties of well studied objects like Mkn 421 and PKS 2155-30. For example, the fractional variability amplitude of the X-ray light curves in Mkn 421 and PKS 2155-304 varies between $\sim 2 \%$ and $\sim 15 \%$ (Edelson et al. 2001; Sembay et al. 2002). These values are comparable to the $f_{\text {rms }}$ values listed in Table 2 for BL Lac. Furthermore, the X-ray power spectrum of PKS 2155-304 and Mkn 421 shows 
a red noise character with a slope of $\sim 2-3$ (Zhang et al. 2002; Kataoka et al. 2001), consistent with the slope of the optical power spectrum of BL Lac. Asymmetric CCFs, with no measurable time lags, similar to the CCFs shown in Fig. 7, have also been observed in PKS 2155-304 and Mkn 421 (Edelson et al. 2001; Sembay et al. 2002). Finally, there are similarities between the X-ray spectral variations observed in Mkn 421 and PKS 2155-304, and the optical band spectral variability of BL Lac as well. Fossati et al. (2000) find that, in the case of Mkn 421, the spectral slope at $5 \mathrm{keV}$ decreases (i.e. the spectrum flattens) as the source flux increases (Fig. 4a in their paper). Their result is based on Beppo-Sax observations in late April/early May 1997, during which no individual flares could be observed. Zhang et al. (2002) also find a similar behaviour between the spectral slope at $0.5 \mathrm{keV}$ and the source flux in PKS 2155-304, again during observations with no obvious, single flares (see for example the spectral slope vs flux plot of the "1997 \#3" data-set in Fig. 11 of their paper). However, clear clockwise and anti-clockwise "loop-like" variations of the spectral slope with respect to the source flux have also been observed in these two X-ray bright BL Lacs. During the April 1998 Beppo-Sax observations of Mkn 421, the hard Xray spectral slope varied with respect to the source flux following an "anti-clockwise" loop (Fossati et al. 2000). Similar loops with a well defined, quasi-circular form have also observed in PKS 2155-304 (Zhang et al. 2002), mainly during observations dominated by well sampled, individual flares. In our case, single flares dominate the observed light curves only during the July 28, 1999 and (mainly) the July 06, 2001 observations. Although in the first case the spectral variations follow the normal "flux increase - spectrum flattening" pattern, it is possible that during the July 06, 2001 observations the variation of the $\alpha_{B I}$ as a function of the $B$ band flux follows an anti- clockwise path during the rising and decaying parts of the flare (see middle panel in Fig. 6). Due to the large errors, we cannot be certain about the reality of this loop-like variation. Longer and better sampled observations (with the collaboration of more than one telescope) are necessary in order to detect and investigate the spectral variations during individual flares and confirm the existence of loop-like variations in the optical band "spectral slope vs. flux" plots of BL Lac.

The similarity in the properties of the fast X-ray variations of Mkn 421 and PKS 2155-304 and optical variations of BL Lac, may be due to same physical reason. The multifrequency spectral energy distribution of BL Lac shows a peak at $\sim 10^{14.5} \mathrm{~Hz}$ while the peak of the synchrotron emission in the other two objects is located at higher frequencies (e.g. Sambruna et al. 1996). Therefore, the optical and X-ray bands correspond to frequencies that are located around/above the peak of the spectral energy distribution of BL Lac on one hand, and Mkn 421 and PKS 2155-30, on the other. This fact, together with the similarity in their fast variability properties imply that the intra-night variations in BL Lac are probably a direct result of the acceleration/cooling mechanism of relativistic electrons which represent the highest energy tail of the synchrotron component, as is generally accepted for the rapid Xray variations in the other two sources. In this case, by studying the optical, rapid variations of BL Lac we can obtain useful information on the acceleration $\left(t_{\text {acc }}\right)$ and cooling $\left(t_{\text {cool }}\right)$ time scales of the most energetic electrons.

Our results suggest that the acceleration process of the energetic particles does not dominate the observed variations. Since the acceleration time scale should be shorter for lower energy particles, we should expect the lower energy (i.e the $I$ band) light curves to show steeper rising phases than the $B$ band light curves. However, we observe the opposite effect.

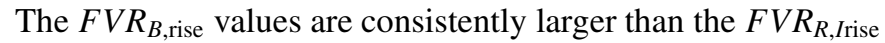
values. Therefore, the $B$ band light curves rise faster than $I$ band light curves. Furthermore, the asymmetry in the CCFs towards positive lags is opposite to what we would expect if the emission propagates from lower to higher energy. We conclude that our observations imply that the injection of radiating electrons in the optical emitting region of BL Lac is almost instantaneous.

In this case emission should propagate from higher (i.e. the $B$ band) to lower energy (the $I$ band) and the higher energy should lead the lower energy photons. This is consistent with the results from the cross-correlation analysis. Although in most cases we do not detect a significant delay (except for the July 5, 2001 observations, see below), the CCF asymmetry towards positive lags implies complex delays between long period components in the two light curves, in the expected direction. If the $B$ band variations during the July 05, 2001 observations do lead the $I$ band variations with $k_{\max } \sim 0.2 \mathrm{hrs}$, then we can obtain an estimate of the magnetic field strength in the source. Assuming that the delay is due to synchrotron losses of the high energy electrons, i.e. $k_{\max }=t_{\text {cool }}(I)-t_{\text {cool }}(B)$, we can use the following formula (e.g. Chiappetti et al. 1999),

$B \delta^{1 / 3} \sim 300\left(\frac{1+z}{v_{I}}\right)^{1 / 3}\left[\frac{1-\left(v_{I} / v_{B}\right)^{1 / 2}}{k_{\max }}\right]^{2 / 3}$,

to estimate $B$ (in $\mathrm{G}$, when frequencies are given in units of $\left.10^{17} \mathrm{~Hz}\right)$. Using $k_{\max }=1.7 \times 10^{4} \mathrm{~s}(=0.2 \mathrm{hrs}), z=0.0688$, and $v_{B}=0.0068, v_{I}=0.0034$ (in units of $10^{17} \mathrm{~Hz}$ ), we find that $B \delta^{1 / 3} \sim 1.3 \mathrm{G}$, or $B \sim 0.4-0.6 \mathrm{G}$, assuming that $10<\delta<30$ (e.g. Vermeulen \& Cohen 1994).

The fact that the rising/decaying time scales of the fully resolved flare during the July 6, 2001 observations are comparable and roughly similar to plateau state duration, bears interesting consequences. If $t_{\text {cool }}<R / c$ (where $R$ is the source radius), the energetic particles would reach equilibrium very fast. The corresponding synchrotron emission should be switched on and off for a short time and, due to light travel effects, after a short period (controlled by the short injection time scale, $t_{\text {inj }}$ ) the observer should see a constant flux, produced by a single "switched on" slice running across the source (Chiaberge \& Ghisellini 1999). After the last region of the source is switched on and off, a fast decline, controlled by $t_{\text {cool }}$, should be observed. Therefore, when both $t_{\text {inj }}$ and $t_{\text {cool }}$ are much smaller than $R / c$, we expect to observe a plateau phase which will last much longer than the rise and decay phase of the flare. The fact that the July 6, 2001 flare is symmetric, with rising/decaying/plateau phases which last roughly the same period, implies that $t_{\text {cool }}$ is not much smaller than $R / c$. In this case, we expect the flux to increase as the observer receives 
photons from an increasing volume. At time $t \sim t_{\text {cool }}$, the parts of the source that were seen first will stop emitting, and the flux will remain $\sim$ constant as new parts of the source start to switch on while the parts closer to the observer are switched off. After $t \sim R / c$, the flux will start decreasing, as the whole source volume has been activated, and the front parts of the source keep switching off.

The hypothesis of a jet perturbation with $t_{\text {inj }}<R / c$ and $t_{\text {cool }} \sim R / c$, can also explain the observed spectral variations. As emission propagates from higher to lower frequencies, it takes time for the higher energy electrons to cool and start emitting at lower frequencies (i.e. in the $I$ band). If the increase in the $B$ band emitting volume is faster than $t_{\text {cool }}$, then the higher energy light curves will rise steeper than the lower energy light curves. As a result, as the $B$ band flux rises, the observed spectrum will flatten systematically. The flattest spectral index will correspond to the maximum $B$ band flux, when the $B$ band emitting region has reached its maximum size, and the volume of the $I$ band emitting region is still increasing. Furthermore, since $t_{\text {cool }}$ is faster for the $B$ band than the $I$ band emitting electrons, the decaying phases of the light curves are expected to be steeper in the $B$ band, as observed. At the same time, the spectrum will become "redder" as the $B$ band flux decreases.

\section{Conclusions}

We have observed BL Lac in three bands, namely $B, R$, and $I$, for 2 nights in July, 1999 and 3 nights in July, 2001. On average, each light curve lasts for $\sim 6$ hours. There are $\sim 40$ points in each of them, almost evenly spaced, with an average sampling interval of $\sim 0.1 \mathrm{hrs}$. Because of the dense sampling and the availability of light curves in three bands, we were able to study in detail the intra-night flux and spectral variations of the source. Our results can be summarized as follows:

1) The source is highly variable in all bands. The variations are smooth, showing rising/decaying phases which, in some cases, last longer than the length of each individual light curve. We have also detected 3 "flare-like" events. In particular, during the July 6, 2001 observation, we observed the whole cycle of a flare which appears symmetric, with a plateau, and lasted for $\sim 3$ hours (in all bands). In general, the rising time scales are comparable to the decaying time scales within each band. However, these time scales are shorter in the $B$ than the other two band light curves.

2) The variability amplitude decreases from $\sim 6.5 \%$ in the $B$ band, to $\sim 5.5 \%$ and $\sim 5 \%$ in the $R$, and $I$ band light curves, respectively. The average, optical power spectrum of the source has a red noise character, with a slope of $\sim-2$ in the frequency range between $\left[5.5(\mathrm{hrs})^{-1}-15(\mathrm{~min})^{-1}\right]$.

3) The light curves in the three bands are well correlated. The variations occur almost simultaneously in all of them, in the sense that the delay between the $I$ and $B$ band variations is smaller than $\sim \pm 0.4 \mathrm{hrs}$. However, we also find that during the July 5, 2001 observation, there is a $95 \%$ probability that the $I$ band light curve variations are delayed with respect to the $B$ band variations by $\sim 0.2$ hrs. Furthermore, most of the CCFs are significantly asymmetric, implying complex delays of the $I$ band variations, in all cases.
4) Finally, the source shows significant intra-night spectral slope variations. These variations are associated with the source flux, in the sense that the spectrum becomes "bluer" as the flux increases. The flattest spectral slope corresponds to the maximum $B$ band flux. The rate of the spectral slope changes is different for different rising/decaying parts of the light curves.

Assuming that the variations are caused by perturbations which activate the jet, the observation of variations with different duration implies that the perturbations affect different regions of the jet. The fact that the rising time scales are steeper in the $B$ band light curves and the CCF asymmetry towards positive lags imply that the injection time scales are very short (i.e. shorter than our average sampling rate which is $\sim 3-6 \mathrm{~min}$ ). These results, together with the observed spectral variability pattern, imply that the observed variations are governed by the cooling time scale of the relativistic particles and the light crossing time scale. The detection of symmetric flares with a plateau state implies that these time scales are comparable. Finally, the detection of a soft lag in one of the observations, allows us to obtain an estimate of the magnetic field strength, $B \sim 0.5 \mathrm{G}$.

We believe that our results demonstrate that well sampled, multi-band optical, intra-night observations of BL Lac objects, whose peak of the emitted power is at $\mathrm{mm} / \mathrm{IR}$ wavelengths so that the optical emission corresponds to the emission from the most energetic, synchrotron emitting electrons in the jet, will offer us important clues on the acceleration and cooling mechanism of these particles. Since the injection and cooling times of the particles are very short, light curves with an average sampling of no more than a few minutes are necessary to this end.

Acknowledgements. We would like to thank E. Pian, the referee, for helpful comments. Skinakas Observatory is a collaborative project of the University of Crete, the Foundation for Research and TechnologyHellas, and the Max-Planck-Institut für extraterrestrische Physik.

\section{References}

Cardelli, J. A., Clayton, G. C., \& Mathis, J. S. 1989, ApJ, 345, 245

Chiaberge, M., \& Ghisellini, G. 1999, MNRAS, 306, 551

Chiappetti, L., Maraschi, L., Tavecchio, F., et al. 1999, ApJ, 521, 552

Clements, S. D., \& Carini, M. D. 2001, AJ, 121, 90

Corbett, E. A., Robinson, A., Axon, D. J., et al. 1996, MNRAS, 281, 737

Edelson, R. A., \& Krolik, J. H. 1988, ApJ, 333, 646

Edelson, R., Griffiths, G., Markowitz, A., et al. 2001, ApJ, 554, 274

Fiorucci, M., \& Tosti, G. 1996, A\&AS, 117, 475

Fossati, G., Maraschi, L., Celotti, A., Comastri, A., \& Ghisellini, G. 1998, MNRAS, 299, 433

Fossati, G., Celotti, A., Chiaberge, M., et al. 2000, ApJ, 541, 166

Fukugita, M., Shimasaku, K., \& Ichikawa, T. 1995, PASP, 107, 945

Ghisellini, G., Celotti, A., Fossati, G., Maraschi, L., \& Comastri, A. 1998, MNRAS, 301, 451

Ghosh, K. K., Ramsey, B. D., Sadun, A. C., Soundararajaperumal, S., \& Wang, J. 2000, ApJ, 537, 638

Kataoka, J., Takahashi, T., Wagner, S. J., et al. 2001, ApJ, 560, 659

Matsumoto, K., Kato, T., Nogami, D., et al. 1999, PASJ, 51, 253

Miller, H. R., Carini, M. T., \& Goodrich, B. D. 1989, Nature, 337, 627 
Nandra, K., George, I. M., Mushotzky, R. F., Turner, T. J., \& Yaqoob,

T. 1997, ApJ, 476, 70

Nesci, R., Maesano, M., Massaro, E., et al. 1998, A\&A, 332, L1

Papadakis, I. E., \& Lawrence, A. 1993, MNRAS, 261, 612

Pian, E., Falomon, R., Hartman, R. C., et al. 2002, A\&A, 392, 407

Peterson, B. M., Wanders, I., Horne, K., et al. 1998, PASP, 110, 660

Racine, R. 1970, ApJ, 159, 99L

Ravasio, M., Tagliaferri, G., Ghisellini, G., et al. 2002, A\&A, 383, 763

Ryter, C. E. 1996, Ap\&SS, 236, 285

Sambruna, R., Maraschi, L., \& Urry, M. C. 1996, ApJ, 463, 444

Scarpa, R., Urry, C. M., Falomo, R., Pesce, J. E., \& Treves, A. 2000, ApJ, 532, 740

Schlegel, D. J., Finkbeiner, D. P., \& Davis, M. 1998, ApJ, 500, 525
Sembay, S., Edelson, R, Markowitz, A., Griffiths, R. G., \& Turner, M. J. L. 2002, ApJ, 574, 634

Smith, P. S., Balonek, T. J., Heckert, P. A., Elston, R., \& Schmidt, G. D. 1985 , AJ, 90, 1184

Speziali, R., \& Natali, G. 1998, A\&A, 339, 382

Urry, C. M., Sambruna, R. M., Worrall, D. M., et al. 1996, ApJ, 463, 424

Vermeulen, R. C., \& Cohen, M. H. 1994, ApJ, 430, 467

Vermeulen, R. C., Ogle, P. M., Tran, H. D., et al. 1995, ApJ, 452, 5L

Villata, M., Raiteri, C. M., Kurtanidze, O. M., et al. 2002, A\&A, 390, 407

Zhang, Y. H., Treves, A., Celotti, A., et al. 2002, ApJ, 572, 762 